\title{
Symptoms reported by gastrointestinal stromal tumour (GIST) patients on imatinib treatment: combining questionnaire and forum data
}

\author{
Dide den Hollander ${ }^{1,2} \cdot$ Anne R. Dirkson ${ }^{3}$. Suzan Verberne ${ }^{3} \cdot$ Wessel Kraaij $^{3} \cdot$ Gerard van Oortmerssen $^{3,4}$. \\ Hans Gelderblom ${ }^{5} \cdot$ Astrid Oosten $^{6} \cdot$ Anna K. L. Reyners $^{7} \cdot$ Neeltje Steeghs $^{1}$ • Winette T. A. van der Graaf ${ }^{1,6}$. \\ Ingrid M. E. Desar ${ }^{2} \cdot$ Olga Husson $^{1,8,9}$
}

Received: 1 December 2021 / Accepted: 19 February 2022 / Published online: 2 March 2022

(c) The Author(s) 2022

\begin{abstract}
Purpose Treatment with the tyrosine kinase inhibitor (TKI) imatinib in patients with gastrointestinal stromal tumours (GIST) causes symptoms that could negatively impact health-related quality of life (HRQoL). Treatment-related symptoms are usually clinician-reported and little is known about patient reports. We used survey and online patient forum data to investigate (1) prevalence of patient-reported symptoms; (2) coverage of symptoms mentioned on the forum by existing HRQoL questionnaires; and (3) priorities of prevalent symptoms in HRQoL assessment.

Methods In the cross-sectional population-based survey study, Dutch GIST patients completed items from the EORTC QLQ-C30 and Symptom-Based Questionnaire (SBQ). In the forum study, machine learning algorithms were used to extract TKI side-effects from English messages on an international online forum for GIST patients. Prevalence of symptoms related to imatinib treatment in both sources was calculated and exploratively compared.

Results Fatigue and muscle pain or cramps were reported most frequently. Seven out of 10 most reported symptoms (i.e. fatigue, muscle pain or cramps, facial swelling, joint pain, skin problems, diarrhoea, and oedema) overlapped between the two sources. Alopecia was frequently mentioned on the forum, but not in the survey. Four out of 10 most reported symptoms on the online forum are covered by the EORTC QLQ-C30. The EORTC-SBQ and EORTC Item Library cover 9 and 10 symptoms, respectively.

Conclusion This first overview of patient-reported imatinib-related symptoms from two data sources helps to determine coverage of items in existing questionnaires, and prioritize HRQoL issues. Combining cancer-generic instruments with treatment-specific item lists will improve future HRQoL assessment in care and research in GIST patients using TKI.
\end{abstract}

Keywords Patient-reported outcomes $\cdot$ Patient forum $\cdot$ Social media mining $\cdot$ Symptom measurement $\cdot$ Tyrosine kinase inhibitors · Gastrointestinal stromal tumours

Olga Husson

olga.husson@icr.ac.uk

1 Department of Medical Oncology, Netherlands Cancer Institute, Amsterdam, the Netherlands

2 Department of Medical Oncology, Radboud University Medical Center, Nijmegen, the Netherlands

3 Leiden Institute of Advanced Computer Science, Leiden University, Leiden, the Netherlands

4 Dutch Patient Platform Sarcomas, Utrecht, the Netherlands

5 Department of Medical Oncology, Leiden University Medical Center, Leiden, the Netherlands
6 Department of Medical Oncology, Erasmus MC Cancer Institute, Erasmus University Medical Center, Rotterdam, the Netherlands

7 Department of Medical Oncology, University Medical Center Groningen, University of Groningen, Groningen, the Netherlands

8 Department of Surgical Oncology, Erasmus Medical Center, Rotterdam, the Netherlands

9 Division of Clinical Studies, Institute of Cancer Research, London, UK 


\section{Introduction}

GISTs represent a rare (10-20 cases per 1,000,000/year) family of mesenchymal tumours arising anywhere along the gastrointestinal tract [1]. Treatment with tyrosine kinase inhibitors (TKIs) improves survival for patients with a gastrointestinal stromal tumour (GIST), both in the adjuvant and palliative setting, but is not without side-effects [2-4]. TKIs are the only effective systemic treatment for high-risk localized and advanced GISTs [5]. Specifically, imatinib has significantly changed the prognosis of non-resectable advanced or metastatic GIST patients: from a median overall survival of 14-18 up to 57 months [6]. TKIs are taken orally on a daily basis until progressive disease. Especially, imatinib is considered to be moderate to well tolerated, at least when compared to conventional chemotherapy [7]. Side-effects are seen in virtually all patients, with the most frequent being (periorbital) oedema, diarrhoea, fatigue, myalgia/musculoskeletal pain, and nausea [8].

Treatment-related side-effects or symptoms have a significant impact on health-related quality of life (HRQoL) and are an important aspect of HRQoL assessment. HRQoL and symptoms can be assessed using patientreported outcome measures (PROMs), providing subjective assessments coming directly from the patient, without interpretation by health care professionals or anyone else [9]. The patient perspective is needed to create a more complete overview of treatment-related symptoms, as previous research has shown a gap between the reporting by clinicians and by patients, with clinicians under-reporting symptoms $[10,11]$. Another resource of patient-reported data is social media, including patient forums, i.e. online communities where patients exchange information and experiences. Social media are increasingly recognized as sources for reports of patient experiences including symptoms [12]. The reports from social media are unselected, unsolicited, and unbiased, and indicate which symptoms have an impact on their health or daily life [13] without the burden of completing questionnaires. Furthermore, its data can also detect emerging issues that may not be mentioned in registration trials or are not covered in existing PROMs [14, 15].

Few studies have investigated patient-reported symptoms in patients with GIST using TKIs. In a qualitative study, 77 different symptoms were reported by GIST patients using TKIs [16]. In another interview study [17], GIST patients with metastatic disease who used imatinib subjectively described the most frequent symptoms as being periorbital oedema, nausea, fatigue, exhaustion, cognitive impairment, muscle pain and cramps, and joint pain. Patients also described the considerable impact of these symptoms on their daily lives, again pointing out the gap between physician-reported side-effects and the lived experiences of patients. Quantitative data are scarce: one study reported severe fatigue in one-third of GIST patients on TKI [18], while another study reported diarrhoea, fatigue, and insomnia [19].

To date, interventional studies in GIST patients often use generic (e.g. Short-Form Health Survey (SF-36) [20]) or cancer-generic (e.g. EORTC QLQ-C30 [21]) PROMs that do not assess symptoms specific to TKIs. To incorporate TKIrelated symptoms in patient-reported outcome measures for GIST patients in future research, more detailed insight into symptom prevalence, relevance, and priority of issues is needed. In the current study, we use two different data sources for patient-reported symptoms, i.e. survey data and data extracted from an online GIST patient forum to examine: (1) the prevalence of symptoms reported by patients; (2) to what extent the issues reported on a patient forum are covered by existing PROMs (i.e. EORTC QLQ-C30 and items from the EORTC Symptom-Based Questionnaire [16]); and (3) the issues that should be prioritized for incorporation in future HRQoL assessment based on the top 10 most prevalent issues.

\section{Methods}

\section{Study design and participants}

A cross-sectional population-based survey study was conducted among patients aged $\geq 18$ years at diagnosis registered in the Netherlands Cancer Registry (NCR) and who had been diagnosed with GIST (according to the ICD10-GM codes C15-20, C26, C48, and C80), between January 1, 2008, and December 31, 2018. Only patients diagnosed within one of the GIST expertise centres (Radboud University Medical Centre [Nijmegen], Erasmus MC Cancer Institute [Rotterdam], Leiden University Medical Centre, The Netherlands Cancer Institute [Amsterdam], and University Medical Centre Groningen) were selected. Patients who had cognitive impairment or were too ill at the time of the study, according to the advice from their (former) treating specialist, or died prior to the start of the study (according to data from the hospital of diagnosis and/or data from the Dutch municipal personal records database) were excluded. The NCR is a population-based registry which is maintained by the Netherlands Comprehensive Cancer Organisation (IKNL) and collects records, including patient, tumour, and treatment characteristics, on all newly diagnosed cancer patients in the Netherlands based on data from the Nationwide Network and Registry of Histo- and Cytopathology (PALGA) in the Netherlands [22]. 
Data from the (at the time) public Facebook group of GIST Support International (GSI) was used to automatically extract symptoms from the messages on the patient forum. GSI is a US-based non-profit corporation founded in 2002 [23]. The main aims of the organization are to connect GIST patients and their families and friends, to provide information, and to stimulate research. Members are encouraged to interact and share ideas and experiences in the online community. The forum was moderated by assigned, experienced GSI members.

Ethical approval for the cross-sectional study was provided by the medical ethical committee of the Radboud University Medical Centre (2019-5888). According to the Dutch law, approval of one ethical committee for questionnaire research is valid for all participating centres. Permission to use data from the Facebook group was given by GSI. Discussions were pseudonymized and messages could not be traced back to individual members. No formal approval was needed for the use of data from the public Facebook group, as the General Data Protection Regulation (GDPR) allows the use of data from publicly accessible forums with justified cause.

\section{Recruitment and data collection}

\section{Survey study}

Eligible patients received an invitation letter from their (ex-) treating physician explaining the goals and procedure of the study. Participants provided informed consent, including permission to link survey data with data from the NCR. Data was collected from September 2020 through June 2021. Survey administration was done within the Patient-Reported Outcomes Following Initial treatment and Long-term Evaluation of Survivorship (PROFILES) registry [24]. PROFILES is a data management system set up in 2009 in the Netherlands for the study of the physical and psychosocial impact of cancer and its treatment. PROFILES contains a large webbased component and is linked directly to clinical data from the NCR. Participants could complete the survey online or on paper upon request.

\section{Forum study}

The English messages from the patient forum were collected on November 1, 2020, and ranged from October 24, 2009, to November 1, 2020. The number of messages was 125,161 in 14,631 conversational threads. A software pipeline was developed to first extract words containing side-effects from each forum message and then to automatically determine which side-effect is being mentioned. These algorithms were trained on data hand-labelled by human annotators. The sensitivity or recall of the extraction of side-effects is
0.739 meaning $73.9 \%$ of the side-effects reported on the forum can be found by the algorithm. The precision is 0.695 , which means that $69.5 \%$ of the side-effects identified by the algorithm are side-effects. The remaining $30.5 \%$ are false positives. The accuracy of automatic labelling of side-effects with SNOMED-CT concepts is 0.645 (i.e. $64.5 \%$ of the side-effects are automatically linked to the correct concept in SNOMED-CT) [25]. Text about imatinib was extracted from the forum data as well and then linked to the symptom mentioned in the message that it was most likely associated with. The methods of sensitivity and accuracy analysis, text-extraction, and linkage of the symptom to imatinib are described in detail elsewhere [25].

\section{Study measures}

Questionnaires and individual items from the EORTC Quality of Life Group (QLG) portfolio were selected as they belong to the most frequently used cancer-specific PROMs worldwide and were developed following well-established guidelines [26]. From the 30-item questionnaire EORTC QLQ-C30, version 3.0 [21], 11 symptom-specific items were evaluated (i.e. dyspnoea, pain, feeling weak, appetite loss, nausea, vomiting, constipation, diarrhoea, fatigue, problems with concentrating, and problems with remembering things). Other symptoms related to TKI use were assessed by 8 additional items from the EORTC SymptomBased Questionnaire (EORTC-SBQ), a 61-item set that was recently developed for patients receiving targeted therapy [16] (i.e. swelling of the face or around the eyes, swelling in any part of the body, muscle aches, pains, or cramps, aches or pains in joints, food and drink tasting different from usual, pain or soreness in mouth, indigestion or heartburn, skin problems). Furthermore, one item about hand-foot syndrome was added from the EORTC Item Library. The items were selected based on prevalence reported in a systematic review of the symptoms associated with TKIs used in the treatment of GIST [27]. One item of own design about the impact of changed physical appearance was added as this was an issue that physicians frequently heard from patients, based on symptoms such as periorbital oedema and hair discolouration.

\section{Statistical analysis}

\section{Survey study}

For analysis, only patients using TKI at the time of study participation were selected. In the case of low numbers of patients using a specific TKI, the results were only exploratively compared and presented separately in Appendix 1 . Prevalence scores for symptoms were determined based on a score of 2 or higher on the 4-point Likert scale being 1 
'not at all', 2 'a little', 3 'quite a bit', and 4 'very much', and represented by numbers and percentages out of the total number of patients taking the specific TKI. All analyses were conducted using SPSS version 25.0 (Statistical Package for Social Sciences, Chicago, IL, USA).

\section{Forum study}

To reduce noise, only side-effects that are mentioned at least five times are included, duplicate side-effects from the same forum message were excluded, and false positives are reduced by excluding cases where no drug is mentioned in the conversational thread [25]. Prevalence of symptoms in the patient forum data was based on how often the symptom was mentioned. Percentages out of the total number of symptoms for each TKI were calculated and reported elsewhere [28].

As a secondary analysis, the 10 most prevalent symptoms for each TKI in the survey study and the forum study were compared based on relative reporting rate. Comparison based on absolute prevalence in the two studies was not possible, because of the difference in measurement.

\section{Results}

\section{Participants}

In the cross-sectional survey study, a total of 521 (former) GIST patients were invited to participate and 328 (response rate 63\%) consented and completed the survey. One hundred seven GIST patients used TKI at the time of study participation: 92 used imatinib, 6 sunitinib, 6 regorafenib, and 3 ripretinib. Based on these numbers, we focused on imatinib treatment for this analysis, and results of the explorative analysis for the other TKIs are included in Appendix 1. Characteristics of patients using imatinib are shown in Table 1. No patient characteristics are available from the forum study.

\section{Prevalence scores}

Prevalence scores for symptoms related to imatinib are shown in Table 2. In the survey study, the three most prevalent patient-reported symptoms for imatinib were fatigue (73\%), muscle pain or cramps $(73 \%)$, and swelling in the face or around the eyes (59\%). In the forum study, for imatinib, the three most prevalent were symptoms fatigue $(8.6 \%)$, nausea $(7.8 \%)$, and cramp $(6.9 \%)$.
Table 1 Patient characteristics from the survey study

\begin{tabular}{|c|c|}
\hline & Imatinib $(n=92)$ \\
\hline Age $($ mean \pm SD) & $66.5 \pm 10.0$ \\
\hline Time since diagnosis in years $($ mean $\pm \mathrm{SD})$ & $6.0 \pm 2.9$ \\
\hline \multicolumn{2}{|l|}{ Sex } \\
\hline Male & 50 \\
\hline Female & 42 \\
\hline Highest formal education & 1 missing \\
\hline Primary school only & $4(4.4 \%)$ \\
\hline High school & $20(22 \%)$ \\
\hline College or university & $67(72.9 \%)$ \\
\hline \multicolumn{2}{|l|}{ Relationship status } \\
\hline Single & 6 \\
\hline Married/relationship & 73 \\
\hline Separated/divorced & 6 \\
\hline Widowed & 7 \\
\hline \multicolumn{2}{|l|}{ Comorbidities $^{\#}$} \\
\hline None & 28 \\
\hline One & 17 \\
\hline Two or more & 47 \\
\hline \multicolumn{2}{|l|}{ Comorbidities (specified) ${ }^{\#}$} \\
\hline Heart disease & 9 \\
\hline Stroke & 2 \\
\hline Hypertension & 21 \\
\hline Lung disease & 7 \\
\hline Diabetes & 7 \\
\hline Ulcer or stomach disease & 3 \\
\hline Kidney disease & 5 \\
\hline Liver disease & 7 \\
\hline Anemia or other blood diseases & 13 \\
\hline Thyroid disease & 4 \\
\hline Depression & 8 \\
\hline Osteoarthrosis & 26 \\
\hline Back pain & 26 \\
\hline Rheumatoid arthritis or other joint inflamma- & 6 \\
\hline tion & 4 \\
\hline Other cancer & \\
\hline
\end{tabular}

\#Assessed using the Self-Administered Comorbidity Questionnaire[29]

\section{Relation between questionnaire and forum symptoms}

Table 3 shows the coverage of the 10 most reported symptoms related to imatinib on the online forum in the EORTC QLQ-C30, the EORTC-SBQ, and the EORTC Item Library. The EORTC QLQ-C30 includes 4 out of 10 most prevalent symptoms on the online forum. The EORTC-SBQ and EORTC Item Library cover 9 and 10 symptoms, respectively.

Finally, the 10 most prevalent symptoms in the survey study and the forum study were compared based on relative reporting rate, indicated as in descending values in Table 4. For imatinib, 7 symptoms overlapped between the two studies. Symptoms from the forum study that were not in the top 10 for imatinib in the survey study 
Table 2 Prevalence* scores for symptoms for imatinib

\begin{tabular}{|c|c|}
\hline Symptoms & Prevalence* $(\%)$ \\
\hline \multicolumn{2}{|l|}{ Survey study $(n=92)$} \\
\hline Fatigue & $66(73)$ \\
\hline Muscle aches, pains, or cramps & $66(73)$ \\
\hline Swelling of the face or around the eyes & $54(59)$ \\
\hline Aches or pains in joints & $48(52)$ \\
\hline Problems with remembering things & $47(52)$ \\
\hline Skin problems (e.g. itchy skin, dry skin, skin discolouration) & $46(50)$ \\
\hline Diarrhoea & $46(50)$ \\
\hline Feeling weak & $38(41)$ \\
\hline Indigestion or heartburn & $37(40)$ \\
\hline Swelling in any part of the body & $35(38)$ \\
\hline Shortness of breath & $31(37)$ \\
\hline Food and drink tasting different from usual & $33(36)$ \\
\hline Pain & $31(34)$ \\
\hline Problems with concentrating & $29(32)$ \\
\hline Problems because of changed appearance & $28(30)$ \\
\hline Appetite loss & $21(23)$ \\
\hline Nausea & $21(23)$ \\
\hline Hand-foot syndrome & $20(22)$ \\
\hline Pain or soreness in mouth & $16(17)$ \\
\hline Constipation & $11(12)$ \\
\hline Vomiting & $5(5)$ \\
\hline \multicolumn{2}{|c|}{$\begin{array}{l}\text { Forum study (10 most prevalent symptoms adapted from https://dashboard-gist-adr.herokuapp.com/ } \\
\text { accessed on July 14, 2021) }\end{array}$} \\
\hline Fatigue & $1181(8.6)$ \\
\hline Nausea & $1062(7.8)$ \\
\hline Cramp & $939(6.9)$ \\
\hline Disorder of skin & $680(5.0)$ \\
\hline Oedema & $544(4.0)$ \\
\hline Pain $^{\mathrm{a}}$ & $524(3.8)$ \\
\hline Alopecia & $466(3.4)$ \\
\hline Altered bowel function ${ }^{\mathrm{b}}$ & $433(3.2)$ \\
\hline Pain in $\operatorname{limb} b^{c}$ & $325(2.4)$ \\
\hline Facial swelling & $235(1.7)$ \\
\hline
\end{tabular}

*For the survey data, prevalence is based on the percentage of patients with this symptom out of the total number of patients taking imatinib. For the forum data, prevalence is based on percentages of each symptom out of the total number of symptoms for imatinib

${ }^{a}$ Includes chronic pain and generalized aches and pains

${ }^{\mathrm{b}}$ Includes constipation and diarrhoea

${ }^{\mathrm{c}}$ Includes any pain in the upper or lower limb, excludes cramp, muscle pain, hand-foot syndrome were nausea, pain, and alopecia (Table 4). Fatigue was the most prevalent symptom both in the survey study and the forum study, but the relative reporting rates for the other symptoms differed. Due to the very low number of patients taking sunitinib, regorafenib, or ripretinib in the survey studies, no formal comparison was made. But explorative analysis showed a similar pattern of overlap between the 10 most prevalent symptoms of the two studies (Appendix $1)$.

\section{Discussion}

This paper describes the use of two sources for patientreported symptom rates outside trials in GIST patients treated with imatinib: surveys and messages from an online patient forum. The most prevalent symptoms in both studies were fatigue and muscle pain or cramps. The EORTC-SBQ and EORTC Item Library cover the majority of symptoms out of the top 10 most prevalent symptoms on the online forum, but coverage by the EORTC QLQ-C30 was limited. 
Table 3 Coverage of symptoms from online forum in questionnaires
Table 4 Ranking in descending values of prevalence of symptoms related to imatinib in survey study and forum study. *Same prevalence $(52 \%)$; \#same prevalence $(50 \%)$

\begin{tabular}{llll}
\hline Symptoms from forum & EORTC QLQ-C30 & EORTC-SBQ & $\begin{array}{l}\text { EORTC } \\
\text { Item } \\
\text { Library }\end{array}$ \\
\hline Fatigue & & & $\mathrm{X}$ \\
Nausea & $\mathrm{X}$ & $\mathrm{X}$ & $\mathrm{X}$ \\
Cramp & $\mathrm{X}$ & $\mathrm{X}$ & $\mathrm{X}$ \\
Disorder of skin & & $\mathrm{X}$ & $\mathrm{X}$ \\
Oedema & & $\mathrm{X}$ & $\mathrm{X}$ \\
Pain & $\mathrm{X}$ & $\mathrm{X}$ & $\mathrm{X}$ \\
Alopecia & & & $\mathrm{X}$ \\
Altered bowel function & $\mathrm{X}$ (diarrhoea, constipation) & $\mathrm{X}$ & $\mathrm{X}$ \\
Pain in limb & & $\mathrm{X}$ & $\mathrm{X}$ \\
Facial swelling & & $\mathrm{X}$ & $\mathrm{X}$ \\
\hline
\end{tabular}

\begin{tabular}{|l|l|l|l|}
\hline Rank & Survey & Rank & Forum \\
\hline 1. & Fatigue & 1. & Fatigue \\
\hline & Muscle aches, pains or cramps & 2. & Nausea \\
\hline 3. & Swelling of face or around the eyes & 3. & Cramp \\
\hline 4. & Aches or pains in joints* & 4. & Disorder of skin \\
\hline & Problems remembering things* & 5. & Oedema \\
\hline 6. & Skin problems\# & 6. & Pain \\
\hline & Diarrhoea\# & 7. & Alopecia \\
\hline 8. & Feeling weak & 8. & Altered bowel function \\
\hline 9. & Indigestion or heart burn & 9. & Pain in limb \\
\hline 10. & Swelling in any part of body (Oedema) & 10. & Facial swelling \\
\hline
\end{tabular}

More than half of the 10 most prevalent symptoms were shared between the two sources, but the relative reporting rate of symptoms differed. The prevalent symptom from the online forum that was not covered by the EORTC-SBQ was alopecia. A similar pattern was found for other TKIs prescribed for GIST in the explorative analysis.

The symptoms found in the survey and the forum study mirror the side-effect profiles of imatinib reported in the registration trials, but relative reporting rates differ for example for muscle cramps [8]. These symptoms occur more frequently over time and may therefore be registered less, or not recognized as adverse drug effects during the initial registration trials. Furthermore, previous work has shown that patients report symptoms earlier and more frequently with worse symptom severity than clinicians [30], and this was particularly the case for muscle cramps and musculoskeletal pain in chronic myeloid leukemia (CML) patients using imatinib [31]. Studies investigating the prevalence of patient-reported symptoms in patients with GIST using TKIs are scarce. Previous studies showed that, similar to our results, severe fatigue is common in GIST patients, especially in those taking TKI [18, 32]. Consequently, fatigue had a negative impact on overall quality of life, functional, psychological, and physical well-being [18]. A study investigating symptom burden with the MD Anderson Symptom Inventory for GISTs (MDASI-GIST) identified the most severe symptoms in GIST patients, including muscle soreness and cramping, fatigue, and general weakness [33], matching the most prevalent symptoms found in our data. Unfortunately, the MDASI-GIST is not validated outside the USA. Symptoms that were most prevalent in our study are also the same as the self-reported side-effects in a qualitative study, such as muscle pain, cramps, and oedema for imatinib [17].

This paper demonstrates that the EORTC portfolio adequately captures what is important to patients on TKI treatment regarding symptoms and HRQoL, although the cancer-generic EORTC QLQ-C30 on its own lacks most treatment-specific symptoms that were reported on the forum. The forum data also reveals side-effects that are not routinely included in PRO-assessment for TKIs, i.e. alopecia. Although it is usually less extensive than in chemotherapy, alopecia is a known adverse effect of TKIs [34, 35] and is more prolonged given the continuous daily dosing schedule. The fact that the reporting rate of alopecia is high on the patient forum indicates that it is an important symptom for patients taking TKIs nonetheless, and can be considered for inclusion HRQoL assessment in future studies.

Differences in relative reporting rate between the two data sources are difficult to interpret, because details on patient characteristics and clinical information were lacking. For example, nausea was ranked higher in the forum 
study for imatinib treatment than in the survey study. Nausea most frequently occurs in the beginning of TKI treatment, and declines over time, e.g. with the use of anti-emetics or changes in dosing schedules [8]. As the survey study included patients who were at least 2.5 years since diagnosis at the time of participation, we hypothesize that the presence of nausea may have already declined whereas patients posting on the forum about nausea may just have started treatment. Furthermore, one might hypothesize that patients who post messages or complete questionnaires experience more symptoms or higher impact on HRQoL than those that do not; however, data on the symptom burden or HRQoL of patients causing them to be active in online cancer communities is scarce. Ector et al. [36] reported that TKI treatment itself and QoL were not associated with a need for more or less information in chronic myeloid leukemia patients. One study found no differences in the use of online support groups for arthritis, fibromyalgia, and breast cancer between patients who post messages and patients who only read messages in case they experienced many or new symptoms [37]. Comparison with a population that was not active on online support groups is not available. The currently used survey study in Dutch GIST patients included evaluation of social media use to investigate differences between patients that use social media to conversate with other patients and those that do not. Analysis of these data is currently ongoing.

Some limitations need to be taken into consideration. First, online forum data and questionnaire data are unavoidably subject to sample bias [38,39] and responder bias, respectively. However, as no background information is available for the posters on the online forum, we cannot assess bias in the current analysis. Furthermore, we have no data on which and how many symptoms were reported by family members of GIST patients who also had access to the forum. In recent years, the use of online support groups by family members was not significantly different from cancer survivors [40], which could also apply to our forum data. Assessment of responder bias in the survey study was also not possible for the subgroup of patients using TKI included in the current analysis, because information about TKI treatment was not available for the non-responder population. Second, a formal comparison of symptom prevalence and prioritization between the two datasets was not possible because of the difference in measurement. The survey study only assessed a limited number of predefined symptoms, whereas the forum study used uncensored, unsolicited reports resulting in a larger number of different symptoms [25]. Prevalence rates were also calculated differently from the two sources, in which methods for extraction of symptoms and linkage to TKI from the online forum could also have induced false positives, e.g. by extracting text that in fact did not refer to a symptom or linkage of a symptom to the wrong TKI [25]. Additionally, patients might post about the same symptom more than once, which could not be assessed without assessing user names and breaching privacy, causing a skewed distribution in the actual frequency and relative reporting rate of the symptoms. Third, it remains challenging to distinguish for patients, and therefore for researchers as well, if symptoms are solely related to treatment, or to tumour burden or comorbidities [41]. This could be clarified in future studies by asking patients to consider the time of onset, or improvement after dose modification. Fourth, the number of patients taking other TKIs than imatinib was low in the survey study, limiting generalizability. This is probably due to including patients who were at least 2.5 years since diagnosis, selecting patients with a favourable course of disease, and/or response to imatinib. Lastly, insufficient information was available in this study to prioritize symptoms for specific subgroups based on clinical characteristics such as time since the start of TKI treatment and treatment setting (adjuvant or palliative).

This study presents an innovative approach to gain more insight into patient-reported symptoms in GIST patients using TKI. Using automatic extraction of symptoms from an online patient forum and linking them to specific TKIs offers a valuable complementary resource for PRO-data. In addition to interviews with patients and health care professionals that are the primary sources for HRQoL issues in PROMs, forum data may include the perspective of patients who would not be invited or not willing to participate in such interviews. It provides insight into which symptoms are relevant in a large group of patients, which is uncommon for rare cancers, which may help prioritize the selection of HRQoL issues for evaluation (e.g. the high prevalence of muscle cramps in this study). Lastly, forum data raises symptoms or side-effects that are not part of existing PROMs (i.e. alopecia in this study), prompting further investigation whether or not they can be included in PROMs and keeping PROMs up to date. This approach is compatible with the novel flexible strategy for HRQoL assessment by the EORTC QLG, combining existing EORTC questionnaires with add-on symptom questions from the EORTC Item Library [42, 43]. In studies investigating GIST (and possibly other cancer) patients using TKIs, we recommend combining the EORTC QLQC30 (to facilitate comparison of cancer-generic HRQoL issues between studies and other (cancer-)populations) with a selection of symptoms from the EORTC-SBQ and individual items from the EORTC Item Library (for symptoms that are missing in the EORTC-SBQ). In studies where only symptoms or adverse events are of interest, the Patient-Reported Outcomes version of the Common Terminology Criteria for Adverse Events (PRO-CTCAE) can also be used [44]. In clinical practice, symptoms can be selected based on known side-effects from registration trials and clinical experience. Hierarchy in relevance 
may be based on data from patient forums. More sensitive detection and measurement of symptoms and their impact on HRQoL will help improve assessment of treatment outcomes in research and shared decision-making about (dis-)continuation of treatment in clinical practice. In conclusion, this study shows the prevalence of TKI treatment-related symptoms reported by GIST patients in a survey and on an online patient forum in a real-life setting. Frequently reported symptoms were not fully covered by cancer-generic measures, and additional issues were reported on the patient forum. Combining these sources of patient-reported data creates a more comprehensive overview of symptom experience and treatment side-effects in GIST patients and helps improve future HRQoL assessment in care and research.

Supplementary Information The online version contains supplementary material available at https://doi.org/10.1007/s00520-022-06929-3.

Acknowledgements We would like to thank Carla Vlooswijk and Esther Derksen-Peters for their support with the recruitment and data collection through PROFILES in the survey study.

Author contribution $\mathrm{OH}, \mathrm{WvdG}$, and ID conceptualized the study and acquired funding. DdH, AD, SV, WK, GO, WvdG, ID, and OH developed methodology. DdH, HG, AO, AR, NS, WvdG, and ID contributed to patient recruitment. $\mathrm{DdH}$ and $\mathrm{AD}$ collected data and performed analysis. The original draft was prepared by $\mathrm{DdH}$ and $\mathrm{OH}$. All authors reviewed and edited the manuscript. All authors read and approved the final manuscript.

Funding The survey study was partly funded by a research grant from Novartis (grant no. 006.18). The forum study was funded by a research grant from the SIDN fund (grant no. 174054). D. den Hollander is supported by a research grant from the EORTC Quality of Life Group (grant no. 003-2018). O. Husson is supported by a VIDI grant from the Netherlands Organization for Scientific Research (VIDI198.007). These funding sources did not have any involvement in the conduction of this research.

Data availability The datasets generated and/or analysed during the survey study are not publicly available due to them containing information that could compromise research participant consent but are available from the corresponding author on reasonable request. The dataset from the forum study is publicly available via https://dashb oard-gist-adr.herokuapp.com/.

Code availability For the forum study, all technical details of the pipeline are reported in a separate publication [25]. The code will be made available upon publication.

\section{Declarations}

Ethics approval Ethical approval for the cross-sectional study was provided by the medical ethical committee of the Radboud University Medical Centre (2019-5888). According to the Dutch law, approval of one ethical committee for questionnaire research is valid for all participating centres. Permission to use data from the Facebook group was given by GIST Support International. Discussions were pseudonymized and messages could not be traced back to individual members. No for- mal approval was needed for the use of data from the public Facebook group, as the General Data Protection Regulation (GDPR) allows the use of data from publicly accessible forums with justified cause.

Consent to participate In the survey study, participants provided informed consent, including permission to link survey data with data from the NCR. Permission to use data from the Facebook group was given by GIST Support International.

Consent for publication Not applicable.

Conflict of interest The authors declare no competing interests.

Open Access This article is licensed under a Creative Commons Attribution 4.0 International License, which permits use, sharing, adaptation, distribution and reproduction in any medium or format, as long as you give appropriate credit to the original author(s) and the source, provide a link to the Creative Commons licence, and indicate if changes were made. The images or other third party material in this article are included in the article's Creative Commons licence, unless indicated otherwise in a credit line to the material. If material is not included in the article's Creative Commons licence and your intended use is not permitted by statutory regulation or exceeds the permitted use, you will need to obtain permission directly from the copyright holder. To view a copy of this licence, visit http://creativecommons.org/licenses/by/4.0/.

\section{References}

1. Søreide K, Sandvik OM, Søreide JA, Giljaca V, Jureckova A, Bulusu VR (2016) Global epidemiology of gastrointestinal stromal tumours (GIST): a systematic review of population-based cohort studies. Cancer Epidemiol 40:39-46. https://doi.org/10. 1016/j.canep.2015.10.031

2. Casali PG, Le Cesne A, Poveda Velasco A et al (2015) Time to definitive failure to the first tyrosine kinase inhibitor in localized GI stromal tumors treated with imatinib as an adjuvant: a European Organisation for Research and Treatment of Cancer Soft Tissue and Bone Sarcoma Group Intergroup Randomized Trial in Collaboration With the Australasian Gastro-Intestinal Trials Group, UNICANCER, French Sarcoma Group, Italian Sarcoma Group, and Spanish Group for Research on Sarcomas. J Clin Oncol 33(36):4276-4283. https://doi.org/10.1200/jco.2015.62. 4304

3. Demetri GD, von Mehren M, Blanke CD et al (2002) Efficacy and safety of imatinib mesylate in advanced gastrointestinal stromal tumors. N Engl J Med 347(7):472-480. https://doi.org/10.1056/ NEJMoa020461

4. Demetri GD, Garrett CR, Schöffski P et al (2012) Complete longitudinal analyses of the randomized, placebo-controlled, phase III trial of sunitinib in patients with gastrointestinal stromal tumor following imatinib failure. Clin Cancer Res 18(11):3170-3179. https://doi.org/10.1158/1078-0432.Ccr-11-3005

5. D'Amato G, Steinert DM, McAuliffe JC, Trent JC (2005) Update on the biology and therapy of gastrointestinal stromal tumors. Cancer Control 12(1):44-56. https://doi.org/10.1177/1073274805 01200106

6. Blanke CD, Demetri GD, von Mehren M et al (2008) Longterm results from a randomized phase II trial of standard- versus higher-dose imatinib mesylate for patients with unresectable or metastatic gastrointestinal stromal tumors expressing KIT. J Clin Oncol 26(4):620-625. https://doi.org/10.1200/jco.2007.13.4403 
7. Casali PG, Abecassis N, Aro HT et al (2018) Gastrointestinal stromal tumours: ESMO-EURACAN Clinical Practice Guidelines for diagnosis, treatment and follow-up. Ann Oncol 29(Suppl 4):iv68iv78. https://doi.org/10.1093/annonc/mdy095

8. Joensuu H, Trent JC, Reichardt P (2011) Practical management of tyrosine kinase inhibitor-associated side effects in GIST. Cancer Treat Rev 37(1):75-88. https://doi.org/10.1016/j.ctrv.2010.04.008

9. U.S. Department of Health and Human Services FDA for Drug Evaluation and Research (2006) Guidance for industry: patientreported outcome measures: use in medical product development to support labeling claims: draft guidance. Health Qual Life Outcomes 4:79. https://doi.org/10.1186/1477-7525-4-79

10. Atkinson TM, Ryan SJ, Bennett AV et al (2016) The association between clinician-based common terminology criteria for adverse events (CTCAE) and patient-reported outcomes (PRO): a systematic review. Support Care Cancer 24(8):3669-3676. https://doi. org/10.1007/s00520-016-3297-9

11. Di Maio M, Basch E, Bryce J, Perrone F (2016) Patient-reported outcomes in the evaluation of toxicity of anticancer treatments. Nat Rev Clin Oncol 13(5):319-325. https://doi.org/10.1038/nrcli nonc. 2015.222

12. Seale C, Charteris-Black J, MacFarlane A, McPherson A (2010) Interviews and internet forums: a comparison of two sources of qualitative data. Qual Health Res 20(5):595-606. https://doi.org/ $10.1177 / 1049732309354094$

13. Robinson KM (2001) Unsolicited narratives from the Internet: a rich source of qualitative data. Qual Health Res 11(5):706-714. https://doi.org/10.1177/104973201129119398

14. Golder S, Norman G, Loke YK (2015) Systematic review on the prevalence, frequency and comparative value of adverse events data in social media. Br J Clin Pharmacol 80(4):878-888. https:// doi.org/10.1111/bcp.12746

15. Tapi Nzali MD, Bringay S, Lavergne C, Mollevi C, Opitz T (2017) What patients can tell us: topic analysis for social media on breast cancer. JMIR Med Inform 5(3):e23. https://doi.org/10.2196/medin form. 7779

16. Sodergren SC, Wheelwright SJ, Fitzsimmons D et al (2021) Developing symptom lists for people with cancer treated with targeted therapies. Target Oncol 16(1):95-107. https://doi.org/10. 1007/s11523-020-00769-z

17. Fauske L, Hompland I, Lorem G, Bondevik H, Bruland OS (2019) Perspectives on treatment side effects in patients with metastatic gastrointestinal stromal tumour: a qualitative study. Clin Sarcoma Res 9:6. https://doi.org/10.1186/s13569-019-0116-3

18. Poort H, van der Graaf WT, Tielen R et al (2016) Prevalence, impact, and correlates of severe fatigue in patients with gastrointestinal stromal tumors. J Pain Symptom Manage 52(2):265-271. https://doi.org/10.1016/j.jpainsymman.2016.02.019

19. Custers JA, Tielen R, Prins JB, de Wilt JH, Gielissen MF, van der Graaf WT (2015) Fear of progression in patients with gastrointestinal stromal tumors (GIST): is extended lifetime related to the Sword of Damocles? Acta Oncol 54(8):1202-1208. https://doi. org/10.3109/0284186x.2014.1003960

20. Ware JE Jr, Sherbourne CD (1992) The MOS 36-item short-form health survey (SF-36). I. Conceptual framework and item selection. Med Care 30(6):473-483

21. Aaronson NK, Ahmedzai S, Bergman B et al (1993) The European Organization for Research and Treatment of Cancer QLQ-C30: a quality-of-life instrument for use in international clinical trials in oncology. J Natl Cancer Inst 85(5):365-376. https://doi.org/10. 1093/jnci/85.5.365

22. Casparie M, Tiebosch AT, Burger G et al (2007) Pathology databanking and biobanking in The Netherlands, a central role for PALGA, the nationwide histopathology and cytopathology data network and archive. Cell Oncol 29(1):19-24. https://doi.org/10. $1155 / 2007 / 971816$
23. GIST Support International (2002) https://www.gistsupport.org/ Accessed on 7 July 2021

24. van de Poll-Franse LV, Horevoorts N, van Eenbergen M et al (2011) The Patient Reported Outcomes Following Initial treatment and Long term Evaluation of Survivorship registry: scope, rationale and design of an infrastructure for the study of physical and psychosocial outcomes in cancer survivorship cohorts. Eur J Cancer 47(14):2188-2194. https://doi.org/10.1016/j.ejca.2011.04. 034

25. Dirkson AR, Verberne S, Kraaij W, van Oortmerssen G, Gelderblom H (2021) Automatic extraction of patient-reported adverse drug events from a GIST patient forum. Connective Tissue Oncology Society Annual Meeting (27):P059

26. Johnson CD, Aaronson NK, Blazeby J et al (2011) Guidelines for Developing Questionnaire Modules 4th Edition. European Organisation for Research and Treatment of Cancer. https:// www.eortc.org/app/uploads/sites/2/2018/02/guidelines_for_ developing_questionnaire-_final.pdf. Accessed on 8 Jan 2020

27. Sodergren SC, White A, Efficace F et al (2014) Systematic review of the side effects associated with tyrosine kinase inhibitors used in the treatment of gastrointestinal stromal tumours on behalf of the EORTC Quality of Life Group. Crit Rev Oncol Hematol 91(1):35-46. https://doi.org/10.1016/j.critrevonc.2014. 01.002

28. Dirkson AR (2021) Automatic extraction of patient-reported adverse drug events from a GIST patient forum. Heroku. https:// dashboard-gist-adr.herokuapp.com/. Accessed on 14 July 2021

29. Sangha O, Stucki G, Liang MH, Fossel AH, Katz JN (2003) The Self-Administered Comorbidity Questionnaire: a new method to assess comorbidity for clinical and health services research. Arthritis Rheum 49(2):156-163. https://doi.org/10.1002/art.10993

30. Basch E, Jia X, Heller G et al (2009) Adverse symptom event reporting by patients vs clinicians: relationships with clinical outcomes. J Natl Cancer Inst 101(23):1624-1632. https://doi.org/10. 1093/jnci/djp386

31. Efficace F, Rosti G, Aaronson N et al (2014) Patient- versus physician-reporting of symptoms and health status in chronic myeloid leukemia. Haematologica 99(4):788-793. https://doi.org/10.3324/ haematol.2013.093724

32. Carbajal-López EB, Juárez-García DM, Espinoza-Velazco A, Calderillo-Ruiz G (2020) Internet-delivered cognitive behavioral therapy and psychoeducation program for patients with gastrointestinal stromal tumors. J Cancer Educ. https://doi.org/10.1007/ s13187-020-01866-3

33. Williams LA, Araujo DM, Shi Q et al (2014) Symptoms in gastrointestinal stromal tumors. J Clin Oncol 32(5s). https://doi.org/ 10.1200/jco.2014.32.15_suppl.9637

34. Mariani S, Abruzzese E, Basciani S et al (2011) Reversible hair depigmentation in a patient treated with imatinib. Leuk Res 35(6):e64-66. https://doi.org/10.1016/j.leukres.2010.11.028

35. Macdonald JB, Macdonald B, Golitz LE, LoRusso P, Sekulic A (2015) Cutaneous adverse effects of targeted therapies: part I: inhibitors of the cellular membrane. J Am Acad Dermatol 72(2): 203-218; quiz 219-220. https://doi.org/10.1016/j.jaad.2014.07. 032

36. Ector GI, Verweij L, Hermens RP, Blijlevens NM (2021) Filling the gaps of patient information needs and information perception in chronic myeloid leukemia with the patient-physician co-produced web-based platform CMyLife. Patient Educ Couns. https:// doi.org/10.1016/j.pec.2021.06.025

37. van Uden-Kraan CF, Drossaert CH, Taal E, Seydel ER, van de Laar MA (2008) Self-reported differences in empowerment between lurkers and posters in online patient support groups. J Med Internet Res 10(2):e18. https://doi.org/10.2196/jmir.992

38. Blank G, Lutz C (2017) Representativeness of social media in Great Britain: investigating Facebook, LinkedIn, Twitter, 
Pinterest, Google+, and Instagram. Am Behav Sci 61(7):741-756. https://doi.org/10.1177/0002764217717559

39. Hargittai E (2020) Potential biases in big data: omitted voices on social media. Soc Sci Comput Rev 38(1):10-24. https://doi.org/ $10.1177 / 0894439318788322$

40. Fareed N, Swoboda CM, Jonnalagadda P, Huerta TR (2021) Persistent digital divide in health-related internet use among cancer survivors: findings from the Health Information National Trends Survey, 2003-2018. J Cancer Surviv 15(1):87-98. https://doi.org/ 10.1007/s11764-020-00913-8

41. Kluetz PG, Slagle A, Papadopoulos EJ et al (2016) Focusing on core patient-reported outcomes in cancer clinical trials: symptomatic adverse events, physical function, and disease-related symptoms. Clin Cancer Res 22(7):1553-1558. https://doi.org/10.1158/ 1078-0432.Ccr-15-2035

42. Bottomley A, Reijneveld JC, Koller M, Flechtner H, Tomaszewski KA, Greimel E (2019) Current state of quality of life and patient-reported outcomes research. Eur J Cancer 121:55-63. https://doi.org/10.1016/j.ejca.2019.08.016

43. Kuliś D, Whittaker C, van de Poll-Franse LV et al (2017) The use of the EORTC Item Library to supplement EORTC quality of life instruments. Value in Health ISPOR 20th Annual European Congress. (20):A775

44. Basch E, Reeve BB, Mitchell SA et al (2014) Development of the National Cancer Institute's patient-reported outcomes version of the common terminology criteria for adverse events (PROCTCAE). J Natl Cancer Inst 106 (9):dju244. https://doi.org/10. 1093/jnci/dju244

Publisher's note Springer Nature remains neutral with regard to jurisdictional claims in published maps and institutional affiliations. 